\title{
Cryotherapy effects measured by infrared thermography in elderly people with rheumatoid arthritis
}

\author{
by V. Svaic* and N.Zura**
}

\author{
* University of Applied Health Sciences, Zagreb, Croatia, vieran.svaic@skole.hr \\ ** University Hospital Centre, Zagreb, Croatia, nikolino.zura@zvu.hr
}

\begin{abstract}
Physiological effects of cryotherapy are well known, but vascular reactions of elderly people suffering from rheumatoid arthritis after the ice massage have not yet been researched enough. Ice massage was applied directly to the hands of the subjects of this investigation after which hand pressure, visual analogue scale for pain-intensity and hand temperature was measured. Short term effects of cryotherapy can be seen in temperature reduction combined with higher hand strength and lower level of pain.
\end{abstract}

\section{Introduction}

Rheumatoid arthritis (RA) is a chronic system inflammatory disease of the connective tissue mostly characterized by destructive process in joints [1]. This disease affects $1 \%$ of the population mostly people between 40 and 60 years old, but it can also start earlier in juveniles up to 16 years of age. During progressive RA destruction of the cartilage is accelerated by the accumulation of liquid and inflammatory cells in synovial membrane. Small wrist joints, finger joints, shoulders, knees and ankles are mostly affected by RA. Affected joints are usually swollen and warm. It is not known whether the inflammatory reactions to the microorganisms are responsible for RA, mechanical injury or if it is the primarily autoimmune disease. However, gene factors together with external factors influence the loss of tolerance and appearance of autoimmune responses to own antigens. Morning stiffness, pain and swelling of joints are the usual symptoms, and usually last for about six weeks before the RA is diagnosed. Half of the patients become incompetent to work after 10 years of the disease, with three times higher medical care cost, higher number of hospitalizations and work absence compared to healthy population. $60 \%$ of the patients under 65 years of age are working impaired which is usually due to the poor treatment of the disease [2].

The treatment is directed to slowing down the destruction of joints, deformities, loss of function and improving the quality of life. General treatment measures, functional treatment and medication therapy are used to treat the disease. Most significant in treatment are non-steroid anti-inflammatory drugs, antirheumatic drugs, corticosteroids and immunosuppressive drugs. Physiotherapy and rehabilitation play a big role in improvement of the course of illness. Usual physical treatments are regular exercise, weight control, healthy diet, warm and cold therapy, relaxation. Ice therapy is a common used physiotherapy method whose main goal is the application of low temperatures in treating the affected joints [3]. Direct effect of cooling is lowering of the tissue temperature, which is associated to lower pain, muscle spasm, circulation, inflammatory process, swelling and sensory nerve impulse speed [4]. Ice is usually placed locally on the affected joint to absorb the heat. The primary physiological reaction of cooling is vasoconstriction, which influences lower blood flow through local tissue, after which a reflex vasodilatation occurs. High inflammatory processes together with the higher temperature of the joints influence the destruction of the tissue. Therefore ice therapy can be effective in treating RA patients. Taking into consideration that skin temperature can be a result of deep tissue metabolic activities like circulatory system changes, inflammatory or degenerative process, therefore thermography represents a means for evaluation of bone, muscle, ligament and neurological structures in the human body.

The main goal of this study was to determine skin temperature change in hands after cryotherapy and the short term effect on hand strength and pain level.

\section{Methods}

Nine patients of the ages 59-83 have been analysed during the ice massage therapy. All the patients were hospitalised at the University Hospital Centre in Zagreb, Croatia, at the Department of Physical Therapy and Rehabilitation. The patients were introduced to the research goals and had signed a written consent for participation in the survey. Visual analogue scale for pain-intensity (VAS) 0-10, dynamometry of the hands (DYN) and hand temperature (TEMP) was measured before the treatment, directly after the treatment and fifteen minutes follow up. Quantitative infrared thermography was used to measure hand temperature by the regions of interest of the hand [5]. Measurements have taken place in a small physiotherapy room with the standard room temperature and air humidity. There was no direct influence of the external heat factors such as airflow, air conditioning or similar. Right after entering the room the patients filled in a short questionnaire with general data and VAS level of left and right hand. Period of acclimatization of the participants was 10 minutes in which they were asked to sit still in the room with palms not touching any parts of their 
body or near objects. After the acclimatization period the initial measurements took place. Thermograms of both hands were taken from their dorsal side (fig. 1), and grip strength using a dynamometer was measured for each hand.

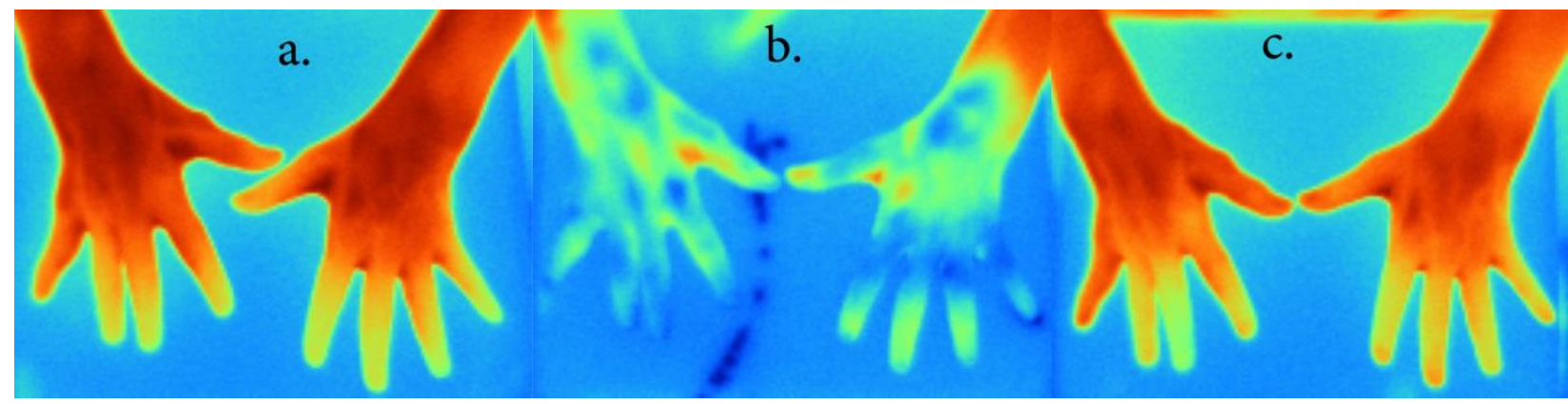

Fig. 1. Thermograms of the hands before ice massage (a), immediately after cryotherapy (b) and 15 minutes after treatment.

Ice massage therapy (ICE) was applied on both hands simultaneously in the average duration on dorsal side 74 \pm 32 seconds and palmar side of the hands $63 \pm 28$ seconds. The duration of application was individual by the participants own feeling of comfort by ice massage. All measurements of VAS, thermography and dynamometry were repeated immediately after the ice treatment and after fifteen minutes follow up during which the patients rested by siting still in the measuring room. Fluke Ti25 infrared camera was used for the purposes of this investigation while for quantitative analysis of the thermograms the authors used Fluke SmartView 3.2 software (Fluke Thermography, USA). The following temperature regions of interest $(\mathrm{ROI})$ were used as variables in this investigation that have been chosen from regions of interest proposed by Ammer [5]: 1. Wrist (ellipse), 2. Thumb (x-section), 3 . Index finger metacarpophalangeal joint (circle), 4. Middle finger (x-section), 5. Middle finger metacarpophalangeal joint (circle). Mean hand temperature was then calculated as an average temperature of all five ROl's.

Statistical analysis was done in SPSS 16 software. Normality of the distributions was tested by skewness and kurtosis, Shapiro-Wilk test and the interpretation of frequency histograms and P-P plots. T-test was used to test the differences between the temperature measurements and Wilcoxon test for nonparametric measurements of VAS and DYN.

\section{Results}

The analysis of the differences, during each of the three measuring periods (initial period before ice massage, immediately after cryotherapy and 15 minutes after treatment), between left and right hand in the measuring variables (VAS, DYN, TEMP) did not show any statistically significant difference. Therefore we proceeded with the further analysis of differences between measuring periods. T-test was used to test those differences and the results can be seen in Table 1. Mean temperature of both hands before ice therapy was $32.69 \pm 2.08{ }^{\circ} \mathrm{C}$. Following the therapy mean temperature dropped to $24.14 \pm 2.26{ }^{\circ} \mathrm{C}$ before it slowly rose during the next fifteen minutes to the mean values of $31.42 \pm 3.22{ }^{\circ} \mathrm{C}$.

Table 1. T-test difference between hand temperatures during three periods of measurement

\begin{tabular}{|c|c|c|c|c|c|c|c|c|}
\hline \multirow{2}{*}{\multicolumn{2}{|c|}{ Paired samples }} & & & & \multicolumn{4}{|c|}{ Paired differences } \\
\hline & & MEAN & $\mathrm{N}$ & SD & MEAN & SD & $\mathrm{t}$ & Sig. \\
\hline \multirow{2}{*}{ 1. PAIR } & TEMPrest & 32.69 & 9 & 2.08 & \multirow{2}{*}{8.55} & \multirow{2}{*}{1.03} & \multirow{2}{*}{24.90} & \multirow{2}{*}{0.00} \\
\hline & TEMPmino & 24.14 & 9 & 2.26 & & & & \\
\hline \multirow{2}{*}{ 2. PAIR } & TEMPrest & 32.69 & 9 & 2.08 & \multirow{2}{*}{1.26} & \multirow{2}{*}{1.36} & \multirow{2}{*}{2.77} & \multirow{2}{*}{0.02} \\
\hline & TEMPmin 15 & 31.42 & 9 & 3.21 & & & & \\
\hline \multirow{2}{*}{ 3. PAIR } & TEMPmin0 & 24.14 & 9 & 2.26 & \multirow{2}{*}{-7.28} & \multirow{2}{*}{1.49} & \multirow{2}{*}{-14.71} & \multirow{2}{*}{0.00} \\
\hline & TEMPmin 15 & 31.42 & 9 & 3.21 & & & & \\
\hline
\end{tabular}

MEAN - average value; $\mathrm{N}$ - number of participants; SD - standard deviation; $\mathrm{t}$ - test value; Sig. - statistical significance; TEMPrest - mean hand temperature during rest period; TEMPmin 0 - mean hand temperature after treatment; TEMPmin 15 - mean hand temperature15 minutes after treatment

Mean drop of hand temperature immediately after the therapy was $-8.55 \pm 1.03{ }^{\circ} \mathrm{C}(t=24.90 ; p=0.00)$. The temperature after 15 minutes of recuperation did not yet rise to the initial level being still significantly lower by $-1.26 \pm$ $1.36{ }^{\circ} \mathrm{C}(\mathrm{t}=2.77 ; \mathrm{p}=0.02)$ then at the initial measurement. This difference is due to a prolonged effect of cooling and the time needed for hand temperature to take initial values. Obviously fifteen minutes was not enough and the re-heating took more time than our investigation was set for. On average the temperature rose for about $0.48{ }^{\circ} \mathrm{C}$ each minute of 
recuperation, with the total of $7.28{ }^{\circ} \mathrm{C}$ during fifteen minutes. The temperature rise was statistically significant ( $\mathrm{t}=-14.71$; $\mathrm{p}=0.00$ ). Nonparametric tests were used for testing the differences in additional two variables (VAS, DYN) between the measurement periods since the variables were not normally distributed. Average values together with standard deviations, minimum and maximum values of those variables can be seen in Table 2.

Table 2. Descriptive statistics of variables VAS and DYN during three periods of measurement

\begin{tabular}{|c|c|c|c|c|c|c|}
\hline Variables & PERIOD & $\mathrm{N}$ & MIN & MAX & MEAN & SD \\
\hline \multirow{3}{*}{ VAS (visual analogue scale) } & Rest & 9 & 1.50 & 8.50 & 6.61 & 2.20 \\
\cline { 2 - 7 } & Min0 & 9 & 0.00 & 7.50 & 4.83 & 2.24 \\
\cline { 2 - 7 } & Min15 & 9 & 1.50 & 7.00 & 5.22 & 1.77 \\
\hline \multirow{3}{*}{ DYN (dynamometry) } & Rest & 9 & 6.65 & 12.70 & 9.09 & 2.17 \\
\cline { 2 - 7 } & Min0 & 9 & 7.80 & 14.10 & 10.51 & 2.58 \\
\cline { 2 - 7 } & Min15 & 9 & 6.40 & 17.95 & 10.33 & 3.43 \\
\hline
\end{tabular}

$\mathrm{N}$ - number of participants; MIN - minimum; MAX - maximum; MEAN - average; SD - standard deviation; VAS - visual analogue scale for pain-intensity; DYN - dynamometry; REST - rest period; Min0 - immediately after treatment; Min15 - fifteen minutes after treatment

Pre-treatment values of grip strength for both hands averaged $9.09 \pm 2.17 \mathrm{~N}$ and immediately after the end of the treatment the values rose to $10.51 \pm 2.58 \mathrm{~N}$ (Table 2). Hand grip strength dropped slightly after fifteen minutes to the values of $10.33 \pm 3.43 \mathrm{~N}$. The statistically significant difference $(Z=-2.19 ; p=0.03)$ was observed between hand grip strength measured by dynamometry during rest period and immediately after ice therapy (Table 3). Interesting finding in this investigation was that hand grip strength after fifteen minutes of recuperation was not statistically different than strength before the therapy $(Z=-0.35 ; p=0.73)$ although mean values were still similarly higher than they were right before the ice treatment.

Table 3. Wilcoxon test of differences between different periods of measurement

\begin{tabular}{|c|c|c|}
\hline & VASrest - VASmin0 & DYNrest - DYNmin0 \\
\hline Z & -2.69 & -2.19 \\
\hline Sig. & 0.01 & 0.03 \\
\hline Z & VASrest - VASmin15 & DYNrest - DYNmin15 \\
\hline Sig. & -2.56 & -1.30 \\
\hline & 0.01 & 0.19 \\
\hline Z & VASmin0 - VASmin15 & DYNmin0 - DYNmin15 \\
\hline Sig. & -0.96 & 0.35 \\
\hline
\end{tabular}

Z - test value; Sig. - statistical significance; VASrest - visual analogue scale during rest period;

VASmin0 - visual analogue scale after treatment; VASmin15 - visual analogue scale 15 minutes after treatment; DYNmir - dynomometry during rest period; DYNmin0 - dynamomtry after treatment; DYNmin 15 - dynamometry 15 minutes after treatment

VAS mean result before the therapy was $6.61 \pm 2.20$ while being statistically significant $(Z=-2.69 ; p=0.01)$ lower after the treatment $4.83 \pm 2.24$. The pain intensity level remained significantly lower $(Z=-2.56 ; p=0.01)$ after the period of fifteen minutes although the values were not as low as they were right after ice therapy $(5.22 \pm 1.77)$. Analysing the results of our investigation it can be seen that all of the testing variables have significantly changed. Hand grip strength has improved for about $15 \%$ while the pain level dropped for approximately $27 \%$. Short term effect of ice therapy treatment can be seen after 15 minutes of recuperation where the pain level still remained lower by $21 \%$. Mean hand temperature after the same period of time was also lower then at the initial stage before the treatment. 
Table 4. Descriptive statistics

\begin{tabular}{|l|c|c|c|c|c|}
\hline \multicolumn{1}{|c|}{ Variables } & $\mathrm{N}$ & MIN & MAX & MEAN & SD \\
\hline Ice therapy duration $($ seconds) & 9 & 45 & 247 & 144.56 & 65.82 \\
\hline Difference TEMPrest-TEMPmin0 $\left({ }^{\circ} \mathrm{C}\right)$ & 9 & 7.22 & 10.35 & 8.55 & 1.03 \\
\hline Difference TEMPrest-TEMPmin15 $\left({ }^{\circ} \mathrm{C}\right)$ & 9 & -0.14 & 3.75 & 1.26 & 1.36 \\
\hline Average TEMPrest $\left({ }^{\circ} \mathrm{C}\right)$ & 9 & 28.68 & 35.71 & 32.69 & 2.08 \\
\hline Average TEMPmin0 $\left({ }^{\circ} \mathrm{C}\right)$ & 9 & 19.60 & 26.30 & 24.14 & 2.26 \\
\hline Average TEMPmin15 $\left({ }^{\circ} \mathrm{C}\right)$ & 9 & 26.31 & 35.33 & 31.42 & 3.21 \\
\hline
\end{tabular}

MEAN - average value; $\mathrm{N}$ - number of participants; SD - standard deviation; MIN - minimum; MAX - maximum; TEMPrest - mean hand temperature during rest period; TEMPmin 0 - mean hand temperature after treatment; TEMPmin15 - mean hand temperature15 minutes after treatment

Correlation was measured between average skin temperatures in three different time periods together with the duration of ice massage (ICE). Descriptive statistics of measured variables can be seen in Table 4 while correlations are shown in Table 5 . Interesting finding was that there was no correlation $(r=0.06 ; p=0.88)$ between duration of the ice therapy to the skin and skin temperature decrease (Diff. TEMPrest-TEMPmin0) immediately after the application. Skin is an organ that reacts to the changes in temperature with vasoconstriction and vasodilatation and by these mechanisms resists high and quick changes. A logical conclusion that the longer application of ice would cause lower temperature of skin was not proven in our investigation. The span of ice therapy application was from minimum 45 seconds to maximum 247 seconds. This was not associated to a higher difference in hand skin temperature from initial period to the period immediately after the therapy.

Table 5. Correlations

\begin{tabular}{|c|c|c|c|c|c|c|c|}
\hline & & $\begin{array}{c}\text { Ice therapy } \\
\text { duration (ICE) }\end{array}$ & $\begin{array}{l}\text { Diff. TEMPrest- } \\
\text { TEMPmin0 }\end{array}$ & $\begin{array}{c}\text { Diff. } \\
\text { TEMPrest- } \\
\text { TEMPmin15 }\end{array}$ & TEMPrest & TEMPmin 0 & TEMPmin 15 \\
\hline \multirow{2}{*}{$\begin{array}{c}\text { Ice therapy } \\
\text { duration (ICE) }\end{array}$} & $r$ & 1.00 & 0.06 & 0.64 & -0.74 & -0.71 & -0.75 \\
\hline & Sig. & & 0.88 & 0.06 & 0.02 & 0.03 & 0.02 \\
\hline \multirow{2}{*}{$\begin{array}{l}\text { Diff. TEMPrest- } \\
\text { TEMPmin0 }\end{array}$} & $r$ & 0.06 & 1.00 & 0.25 & 0.07 & -0.39 & -0.07 \\
\hline & Sig. & 0.88 & & 0.51 & 0.87 & 0.29 & 0.87 \\
\hline \multirow{2}{*}{$\begin{array}{l}\text { Diff. TEMPrest- } \\
\text { TEMPmin15 }\end{array}$} & $r$ & 0.64 & 0.25 & 1.00 & -0.72 & -0.78 & -0.89 \\
\hline & Sig. & 0.06 & 0.51 & & 0.03 & 0.01 & 0.00 \\
\hline \multirow{2}{*}{ TEMPrest } & $r$ & -0.74 & 0.07 & -0.72 & 1.00 & 0.89 & 0.96 \\
\hline & Sig. & 0.02 & 0.87 & 0.03 & & 0.00 & 0.00 \\
\hline \multirow{2}{*}{ TEMPmin 0} & $r$ & -0.71 & -0.39 & -0.78 & 0.89 & 1.00 & 0.91 \\
\hline & Sig. & 0.03 & 0.29 & 0.01 & 0.00 & & 0.00 \\
\hline \multirow{2}{*}{ TEMPmin 15} & $r$ & -0.75 & -0.07 & -0.89 & 0.96 & 0.91 & 1.00 \\
\hline & Sig. & 0.02 & 0.87 & 0.00 & 0.00 & 0.00 & \\
\hline
\end{tabular}

$r$ - Pearson correlation; Sig. - significance; TEMPrest - mean hand temperature during rest period; TEMPmin0 - mean hand temperature after treatment; TEMPmin15 - mean hand temperature15 minutes

Hand skin temperature of our patients was very stable and showed high levels of correlation between initial state, immediately after application of ice and fifteen minutes follow up. On the other hand there was a high variability of initial hand skin temperatures among our participants (minimum $28.68{ }^{\circ} \mathrm{C}$ and maximum $35.71{ }^{\circ} \mathrm{C}$ ). This meant that people with higher resting skin temperature have had higher skin temperature after the ice therapy, and again higher skin temperature after fifteen minutes follow up than those whose initial hand skin temperature was lower. High negative correlations were found between duration of ice massage and the temperature of the hands during all three periods of measurement (TEMPrest $-r=-0.74 ; p=0.02$; TEMPmin0 $-r=-0.71 ; p=0.03$; TEMPmin15 $-r=-0.75 ; p=0.02$ ). This is the evidence that the higher the skin temperature during the initial state, the lower was the duration of ice application. Most probable explanation for this is that the patients feeling of comfort while applying ice to the skin is associated with the initial warmth of their hands. Once the patients start feeling discomfort they usually stop the therapy. 


\section{Conclusion}

According to these findings the authors conclude that ice massage can be effective physiotherapeutic method for lowering pain levels in patients with rheumatoid arthritis together with improvement of function. This effect is instant and can last over 15 minutes during which period other therapeutic methods like kinesitherapy or hand exercise can be done. Also this therapy is very simple and can be done by a patient on his own at any time of the day and whenever he or she needs a relief from pain. Hand grip strength improvement was probably associated to lower level of pain immediately after cryotherapy, therefore hand strength being higher after the therapy. The prolonged effect of higher hand grip strength was not proven in this investigation, although the temperature and pain levels were still lower then at the initial measurement. It can be argued that with a bigger sample of participants the results would differ and that the grip strength would have also been statistically different, but for time being the difference stays insignificant. As it is for the thermography and its value in addressing the temperature changes during ice therapy procedures, it stays obvious that the changes will occur after the treatments since lowering the temperature is the main goal of this type of physiotherapy. It is still interesting to see what is the average drop of the hand temperature after a certain duration of ice therapy and additionally what is the time needed for hand temperature to return to the initial values. Whether this period of recuperation is associated to the inflammatory disease still stays unclear without a control group of patients. This and a lot of other questions offer a prosperous future to infrared thermography in medical use.

\section{REFERENCES}

[1] Čargonja, M., Tomić, R. (2012). Rhumatoid arthritis in elderly. Reumatizam, vol. 59(2), p.p. 197, 2012.

[2] Perković, D. i sur. Clinical approach to the patient with rheumatoid arthritis. Reumarizam, vol. 61(2), p.p. 24-28, 2014.

[3] Agostinucci, J., McLinden, J., Cherry, E. The effect of cryotherapy and exercise on lateral epicondylitis: a controlled randomised study. International Journal of Therapy \& Rehabilitation, vol. 19(11), p.p. 641-650, 2012.

[4] Boland, M., Mulligan, I., Payette, J., Serres, J., O'Hara, R., Maupin, G. A novel cryotherapy compression wrap in the management of acute ankle sprains: potential use for special operators on the battlefield. Journal of Special Operations Medicine, vol. 12(4), p.p.17-23, 2012.

[5] Ammer, K., "The Glamorgan Protocol for recording and evaluation of thermal images of the human body". Thermology international, vol. 18, p.p. 125-144, 2008. 